\title{
Arthroscopic surgery's role in massive tears of the rotator cuff
}

\author{
René Ochoa-Cázares ${ }^{1,2}$, René G. Narváez-Corona ${ }^{1,2}$, and Alejandro González-Parás ${ }^{1,3 *}$ \\ ${ }^{1}$ Arthroscopy an Joint Reconstruction, "Ángeles del Pedregal" Hospital, Mexico City; ${ }^{2}$ Mexican Faculty of Medicine, La Salle University, Campus \\ México City, Mexico City; ${ }^{3}$ Traumatology and Orthopedics Service, "José Eleuterio González" University Hospital, Universidad Autónoma de Nuevo \\ León (UANL), Monterrey, Nuevo Leon. Mexico
}

\begin{abstract}
Advances in arthroscopic surgery have achieved a greater role in the treatment for massive tears of the rotator cuff, whether they are reparable or not. This allows better management for pain, function, and sometimes muscle strength. To choose the best treatment, the surgeon must consider the chief complaint, age, functional stage, anatomy of the lesion, and associated symptoms. We have designed an algorithm that focuses on the treatment options available through arthroscopic surgery, avoiding greater damage of soft tissues, joint preservation, and without precluding other options for treatment such as tendon transfer or reverse shoulder arthroplasty. The treatment options are subacromial debridement, rotator cuff partial repair, patch augmentation, superior capsular repair, and biodegradable subacromial spacer. Each procedure has their own advantages, disadvantages, and indications, which allows us to organize the best treatment option according to the patient's needs.
\end{abstract}

Key words: Shoulder. Rotator cuff. Massive tears. Arthroscopic treatment.

\section{Introduction}

Massive tears of the rotator cuff are described as those which, given their size and retraction, cannot be repaired at their insertion site in the tuberosities despite soft-tissue mobilization and liberation'.

Ogata and Uhthoff described an increase of prevalence of these injuries linked to age, where approximately up to $70 \%$ of patients who were 70 years or older presented injuries of the rotator cuff, from which, $40 \%$ of the ruptures are usually massive $e^{2,3}$. Moreover, observations suggest that there are more recurrent tears in massive ruptures compared to smaller ones ${ }^{4}$.

There are two main groups of patients who present massive ruptures of the rotator cuff, even though these injuries may occur in any age group while performing any level of activity. The first group consists of elderly patients, usually female, with low functional demands, and asymptomatic until a minor trauma causes them discomfort. The other groups consist of younger, more active patients, who begin with pain and a decrease of function after an acute trauma, or present a history of a previous surgery of the rotator cuff, or chronic injury of the latter ${ }^{1}$. Despite the gap in age and the difference in the level of activity, the trigger is usually an injury or trauma of variable degree for both groups.

Loew described the mechanisms that lead to a rotator cuff injury: forced passive rotation, passive traction, or axial compression in a cranioventral or ventromedial direction; direct impact is not considered a cause of rupture ${ }^{2,5}$

\footnotetext{
Correspondence:

*Alejandro González-Parás

E-mail: dralejandrogp@gmail.com

Available online: $18-06-2020$

Date of reception: 18-12-2019

DOI: 10.24875/RMU.20000160

Medicina Universitaria. 2020;22(2):65-72 www.medicinauniversitaria.org

作 (http://creativecommons.org/licenses/by-nc-nd/4.0/).
} 


\section{Biomechanics}

The shoulder becomes biomechanically affected in massive tears due to an increase of internal rotation in posterosuperior ruptures, maximum external rotation in anterosuperior ruptures, and in total rotation range of motion at any abduction angle in cadaver models. Passive internal rotation is affected with the rupture of the tendons in charge of external rotation, like the infraspinatus and teres minor. Subscapularis tears condition weakness in active internal rotation and increase passive external rotation. To accomplish a stable abduction in posterosuperior tears, the forces required by the deltoid and subscapularis are increased. The progression of the tear results in a subluxation of the humeral head due to a loss of coupling forces. The fibers of the injured tendons do not take part in load distribution, thus increasing the tension force in the remaining fibers, which could lead to an increase in the tear. Longitudinal extension of the ruptures also affects the glenohumeral kinematics since it allows the proximal migration of the humeral head. Once this happens, with abduction, there is a pinching of the rotator cuff between the acromion and the humeral head, creating a vicious cycle between an increase in rupture and migration of the humeral head ${ }^{4}$.

\section{Clinical features}

These patients may present a variety of symptoms, ranging from mild discomfort to total limitation of daily activities and severe pain ${ }^{1}$. Other related symptoms may be pain at night, which interrupts sleep, and in some cases pseudoparalysis ${ }^{2}$. Pseudoparalysis is defined as an active anterior flexion of less than $90^{\circ}$ with a normal passive range of motion in the absence of neurological deficits ${ }^{4}$.

During physical examination, an atrophy of scapular muscles in patients with chronic injuries is found. In more severe cases, crackling and hemarthrosis are evident. Patients with posterosuperior disruption present a decrease in abduction, anterior flexion, and active external rotation; this deficiency can result in external rotation lag sign or ERLS, which does not allow overcoming resistance when internal or external rotation of the shoulder is attempted ${ }^{1}$. Moreover, the Hornblower's or Patte's sign can be observed, where the patient is standing and the physician elevates the arm $90^{\circ}$ in the scapular plane and flexes the elbow $90^{\circ}$. After asking the patient to perform a lateral rotation of the shoulder, if the patient presents signs of pain or weakness, then the test is positive ${ }^{6}$. This sign has been described with a sensitivity of $100 \%$ and a specificity of $93 \%$ to identify irreparable injuries of the teres minor ${ }^{1}$.

\section{Classification}

Patte's classification for complete rupture of the rotator cuff is based on retraction in the coronal plane and is mainly used for research work ${ }^{7}$. This classification consists of determining the extent of the injury and its topography on the coronal and sagittal planes. The description of the coronal plane is generally utilized, which is described as Grade 1 for proximal stump near bony insertion; Grade 2 for proximal stump at level of humeral head; and Grade 3 for proximal stump at glenoid level ${ }^{8}$.

Massive injuries can be classified according to their size or the number of tendons involved. There are several classifications for which the criteria of massive rupture vary from author to author. Bateman classifies massive injuries as Grade IV, in which the injury presents a defect of over $5 \mathrm{~cm}$ in arthrography, while Ellman and Gartsman identified massive ruptures as an injury of at least three tendons or two tendons with retractions $>4 \mathrm{~cm}$ in double-contrast arthrography and an MRI of the shoulder $2,9,10$. DeOrio and Cofield classified massive ruptures as those which were $>5 \mathrm{~cm}$ in an anteroposterior or mid-lateral direction, and Gerber described them as those that involve two tendons ${ }^{2,11,12}$. Gerber also commented that tears involving three tendons were less painful than those involving two, and much less painful than the variant that involved an anterosuperior rupture of two tendons ${ }^{12}$.

Anatomical patterns of massive ruptures usually consist of a complete tear of supraspinatus and infraspinatus tendons and teres minor or complete tears of the supraspinatus and subscapular accompanied by injury of the long head of the bicep tendon'.

Magnetic resonance makes definition with a better quality of soft tissues possible, allowing for the identification of ruptures with $100 \%$ sensitivity, as well as an estimation of up to $77 \%$ of the width of an injury, and retractions of up to $63 \%{ }^{1}$. Rotator cuff injuries are observed in the magnetic resonance as a focal area of bright signal, similar to that of the presence of fluid in the tendon. The injury may be filled with articular liquid or granulation tissue, which is observed hyperintensity in T2 images, and in full ruptures extends from the articular surface to the bursal surface of the tendon. The increase of fluid in the subacromial bursa is also a related finding. Complete rupture can allow different degrees of retraction, visible in the resonance ${ }^{13,14}$. 
The amount of fatty infiltration is directly linked to the risk of rerupture and to a poor functional prognosis. Patients who present a high degree of fatty infiltration and muscle atrophy, in addition to a significant elevation of the humeral head toward the acromion, have a greater risk of having irreparable injuries ${ }^{1}$. This fatty infiltration and atrophy occur when there is a deficient muscular contraction in chronic ruptures ${ }^{2}$. Moreover, it has been observed that in massive ruptures, there is traction of the suprascapular nerve, which contributes to the atrophy and streaks of fat ${ }^{4}$. Goutallier et al. classified fatty infiltration according to its relation with the number of muscle fibers in coronal and sagittal T1 images, beginning at Grade 0 with normal muscle; Grade 1 with minimal fatty streaks; Grade 2 where the amount of muscle is equal to the fatty infiltration; Grade 3 with more fatty streaks than muscle; and Grade 4 with total replacement with fat ${ }^{14,15}$. Goutallier et al. originally described fatty infiltration in computerized tomography images, and in some cases, there was fatty infiltration in tendon muscles that did not necessarily have a rupture ${ }^{15}$.

Classifications by Patte and Goutallier have been described to have moderate concordance between observers and ought to be used carefully when deciding on the course of treatment: these classifications are commonly employed to describe preoperative findings in magnetic resonance ${ }^{16}$.

It is important to distinguish between a massive tear, an irreparable tear, and arthropathy of the rotator cuff to choose the right type of treatment. An irreparable injury is accompanied by the characteristics of a massive injury, in addition to static migration of the humeral head upwards, reduction of subacromial space, atrophy, and fatty infiltration of more than $50 \%$ of the muscle and its arthrosic changes. Neer et al. described arthropathy of the rotator cuff as an insufficiency of the cuff, a migration of the humeral head toward proximal and glenohumeral osteoarthritis $6,17,18$. There is degeneration of the glenohumeral articular cartilage and osteoporosis of the humeral head by mechanical and nutritional factors due to instability of the humeral head and leak of synovial fluid, respectively. Eventually, the humeral head collapses producing the complete picture of arthropathy and producing major erosion as a result of the exaggerated loss of articular congruence ${ }^{18}$.

\section{Treatment}

Treatment type depends on the symptoms, age, and functional level of the patient. Moreover, an intact coracoacromial arch, comorbidities of the patient, and the presence or absence of glenohumeral arthrosis are crucial, as well as articular stability ${ }^{1}$.

Among the surgical options, there are subacromial debridement with biceps tenotomy and acromioplasty with or without partial repair ${ }^{1}$, full repair ${ }^{6}$, patch augmentation ${ }^{6,9}$, superior capsular reconstruction ${ }^{20,21}$, and subacromial spacer22,23. Other open major procedures include open repairs, tendon transfer, and reverse arthroplasty of the shoulder'.

Reconstruction in under 3 months is indicated for complete, acute, symptomatic tears of the rotator cuff and must not be delayed to manage conservatively unless the patient refuses surgical management. It has been noted that with time, the defects tend to increase, and asymptomatic patients gradually develop pain ${ }^{2}$.

Management by arthroscopy is more notable with soft tissues than open surgical procedures and today, is considered the golden standard in surgical management for rotator cuff injuries. At present, it is possible to manage from partial tears to massive ones with arthroscopy. Nevertheless, the decision between arthroscopic and open management remains a topic of controversy $^{2}$. Due to muscular retraction and degenerative changes in the muscle, it is recommended that young patients with a confirmed diagnosis of rotator cuff rupture receive surgical management as a first choice ${ }^{2}$.

We have grouped these treatment options into an algorithm at the end of the text, according to the characteristics of the injury and of the patient (Figs. 1 and 2).

\section{Subacromial debridement}

Subacromial debridement is indicated for healthy patients of low functional demand whose main symptom is pain. It shows best results in patients who keep active elevation and descent control of the shoulder, as well as glenohumeral stability. Patients who display functional improvement after subacromial injection are also good candidates ${ }^{4}$.

The advantage of performing it arthroscopically is to avoid injuring the deltoid insertion. Its main objective is to reduce pain and improve range of motion, and muscle strength does not usually change after surgery. The patients should have a competent deltoid and an intact coracoacromial arch and be without rotator cuff pseudoparalysis or arthropathy. It is important that the patient comprehends that the objective of the surgery is to reduce pain, not to improve muscle strength or functionality. It is considered a rescue procedure ${ }^{17}$. 


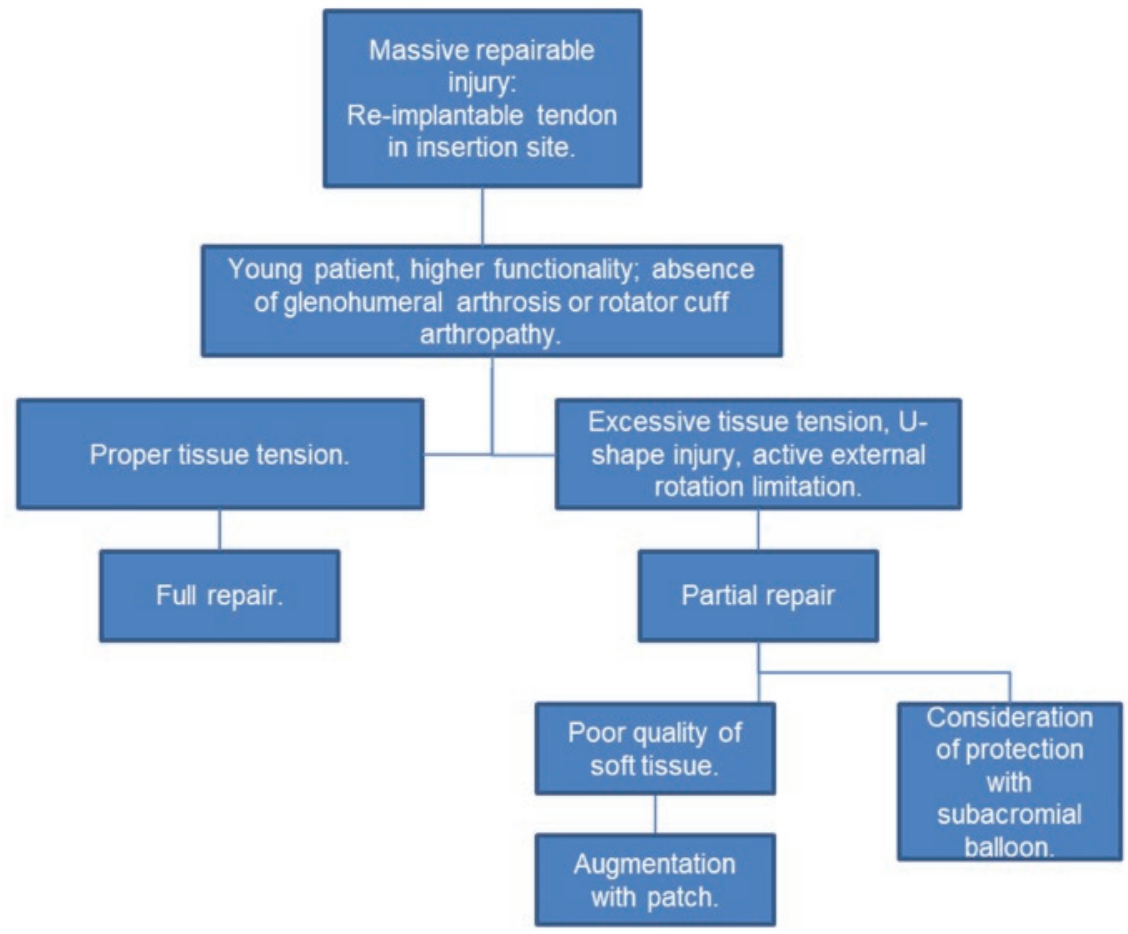

Figure 1. Rotator cuff repair massive rupture management algorithm.

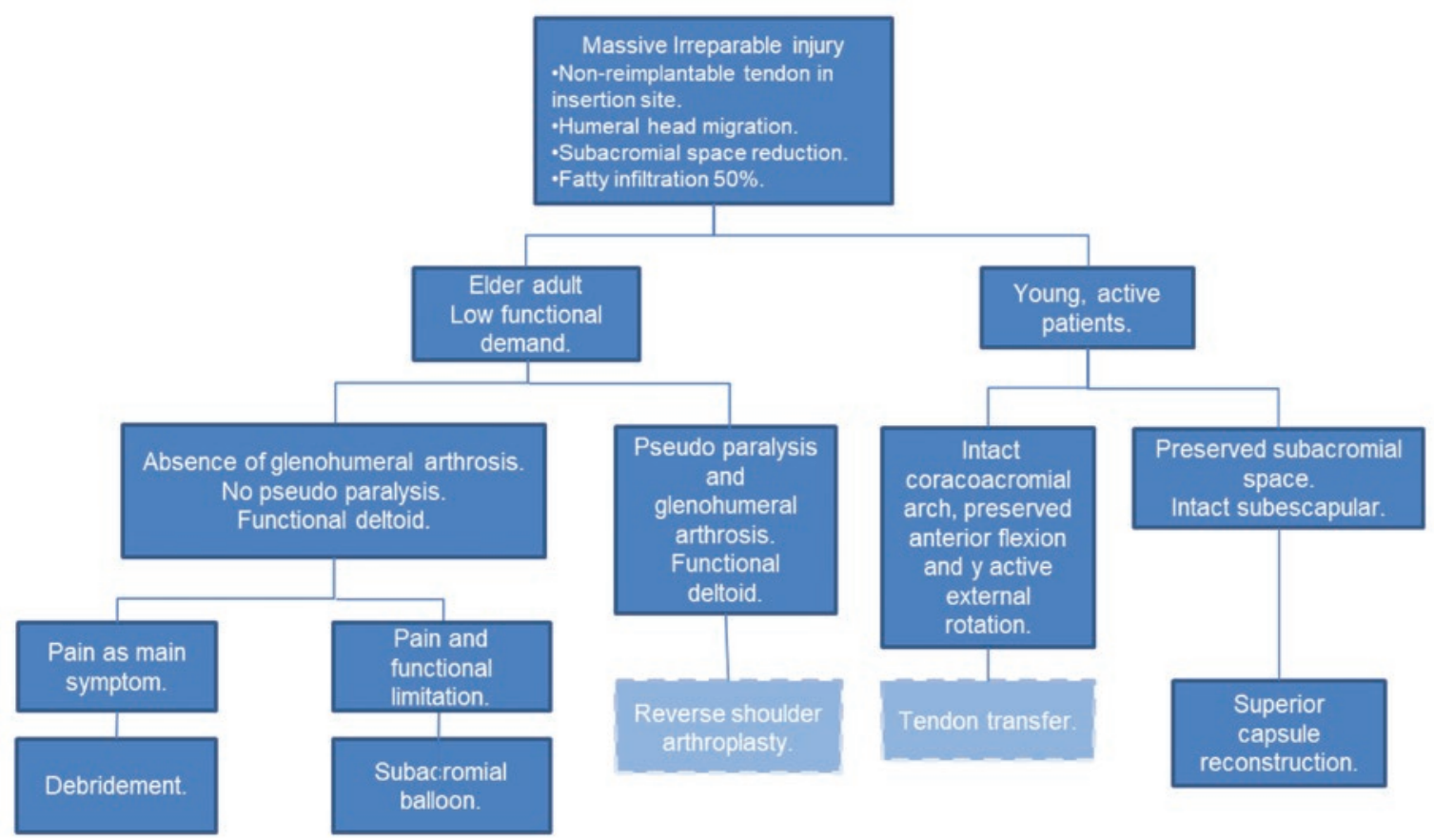

Figure 2. Irreparable massive rotator cuff management algorithm. Non-arthroscopic management alternatives are represented in light blue. 
The steps to be followed during a debridement include systemically locating the areas of pain in the joint through a diagnostic arthroscopy, debridement of the unstable rotator cuff, bursectomy, and resection of scar tissue, release of the rotator cuff and capsulotomy, synovectomy, acromioplasty, tuberculoplasty, and finally tenotomy and tenodesis of the long head of the biceps $^{17}$. Isolated arthroscopic tenodesis and tenotomy of the biceps show effective results for the management of irreparable massive ruptures of the rotator cuff; furthermore, tenotomy is considered in patients older than 50 years of age without heavy lifting activities, whilst tenodesis should be considered for active patients under 40 years of age, with a bone or soft-tissue injury of less than a year of evolution ${ }^{6,24}$. In a retrospective study of 68 patients with irreparable massive ruptures, isolated tenotomy or tenodesis was performed, finding positive results in $78 \%$ of patients. Performing either of these procedures is not recommended in cases with pseudoparalysis or arthropathy of the rotator cuff ${ }^{24}$.

The ideal patient is the one who presents an irreparable injury by size, without arthrosis, who is non-candidate for tendon transfer and with significant pain which does not respond to a conservative management. Other factors which may be considered as a significant factor are the patient's age, as well as his/her coracoacromial arch integrity and an intact deltoid function, manifested as the ability to keep the arm elevated horizontally ${ }^{17}$.

Therefore, we can conclude that arthroscopic debridement is preferable in elderly patients with low functional demand and with irreparable injuries compensated by the action of other muscles. Coracoacromial arch loss is linked to severe failure. Other procedures ought to be taken into consideration for younger patients with greater functionality ${ }^{1}$.

Gartsman found that irreparable ruptures with a compromised subscapular or infraspinous with humeral head migration were predictors of a bad prognosis for management with debridement and according to Boileau, patients with pseudoparalysis will not benefit from debridement ${ }^{17,24,25}$.

After this procedure, an improvement of the anterior flexion of $105-140^{\circ}$ has been described ${ }^{4}$. Moreover, according to different studies, an increase of 34.9-84 points and 66- 91 points in the Constant score has been found, which defines the level of pain and the ability to perform normal every day activities ${ }^{4,17}$. The Constant score consists of two parts, a subjective part in which pain and every day activities are evaluated, and the second part where shoulder strength and mobility are assessed. It is used to evaluate the degree of functionality of the shoulder and helps to determine the degree of improvement ${ }^{26}$. The subjective portion can add up to 35 points, while the objective part up to 65 points, with a total score of 100 points $^{27}$.

\section{Partial repair}

A complete repair after a massive rupture is not always surgically possible due to the quality of the tissue, tendon loss, severe retraction, or increase in tissue tension. Clinical and surgical findings have been compared, discovering that severe impairment, limitation of active external rotation, bad quality of the tendon, large ruptures, and "U"-shaped ruptures can often only be partially repaired ${ }^{4}$. If the tissue does not have adequate tension for a complete repair, it is convenient to perform a partial repair 6 .

The advantages of partial repairs of anterior and posterior portions of injuries without transposition in some patients have been described. This type of repair presents improvements in pain and function. Ellman found that in 19 out of 22 shoulders, no improvements in strength or in range of motion were described after partial arthroscopic repair ${ }^{1,9}$. Burkhart also described improvement of pain and function in patients with stable irreparable injuries in 1 out of 11 shoulders in a study.,28 However, Zvijac et al. have recently described that there is a deterioration in pain reduction, strength, and functional improvement with arthroscopic procedures ${ }^{1,29,30}$.

Contraindications for a repair include advanced degenerative disease of the shoulder, rotator cuff arthropathy, active shoulder infection, or paresis of the subscapular or axillar nerves ${ }^{2}$. The degree of failures in repairs of massive ruptures ranges between 25 and $94 \%{ }^{17}$.

Massive tear repair has been described with a satisfaction rate of $75 \%$, but with a significant reduction of pain compared to postsurgical satisfaction rates in small and medium injuries, which is between $88 \%$ and $95 \%^{2}$.

A study with partial repair with biceps tenodesis, patients had an active elevation in subscapular plane $>120^{\circ}$ without data of delay in external rotation, nor data of degenerative glenohumeral arthrosis. An increase in the ASES score was registered, going from 43 to 79.3 at the end of the study. This score assesses functionality and presence of pain, as well as a reduction in the visual analog scale going from a 6.9 to a 1.9. There were no significant changes in range of motion, 
external or internal rotation. After 5 years, 23 of the 28 patients in the study were found to be satisfied with the results of the surgery. ${ }^{31}$

Furthermore, reports suggest that partial repairs produce abnormal kinematics. Proper restoration of external and internal rotation arches is not accomplished; nevertheless, restoration of the position of the humeral head has been observed to be possible. Studies have not found a significant difference regarding function comparing complete and partial repair groups of the rotator cuff ${ }^{4,32}$.

\section{Patch augmentation}

The purpose of using a frame or a patch helps mechanically by redistributing the loads of the repair and biologically by improving wound healing rate and quality ${ }^{19}$.

It is recommended in active patients with minimal glenohumeral arthrosis. The use of patches for augmentation has been shown to reinforce repair and improve healing potential. It is an alternative to tendon transfers, particularly in those with bad tissue quality ${ }^{4,19}$. Among the utilized materials the non-biodegradable extracellular matrix and degradable synthetic scaffolds stand out, the type of patch utilized tends to have an influence in wound healing ${ }^{4}$.

Biological materials employed are autogenous fascia lata, acellular human dermis, and porcine dermal collagen. Synthetic materials are poly-L-lactic acid, polyester, polypropylene, and polytetrafluorethylene or polyurethane ${ }^{19}$.

Today, there are three types of frames used: xenografts, allografts, and synthetic frames. Xenografts are the least utilized since they do not improve repair and its rerupture rate is similar to that of a repair without augmentation, in addition to a more severe inflammatory reaction and its immunogenic potential. These last couple of situations have decreased after improving sterilization and processing techniques of theses tissues. The advantages of biological frames (auto or allografts) are a result of quick host cell integration, proliferation, migration, and induction of the new tissue. The disadvantages are slower integration, unknown degradation, and variable rates of biocompatibility. Synthetic frames are used because of their mechanical stability and are of a more consistent quality and do not present a risk of transmission of disease or immunogenicity ${ }^{19}$.

After an open or an arthroscopic repair, the patch is placed arthroscopically or by a minimally invasive or open route ${ }^{4}$. Through arthroscopy, its best application is in cases of bad tissue quality for augmentation of massive but repairable injuries. It is not recommended for bypassing irreparable ruptures. In these cases, the use of tendon transfer, superior capsular reconstruction, or the use of reverse shoulder prosthetics are recommended ${ }^{19}$.

The Food and Drug Administration does not authorize the use of augmentation patches for breaches $>1 \mathrm{~cm}$ despite the fact that patches have been used in the past to bypass breaches following retractions of the rotator cuff from its tendon insertion ${ }^{4,19}$.

Gupta conducted a study where 24 patients with massive ruptures of the rotator cuff underwent interposition repair using acellular human dermal allografts. After 3 years of follow-up, an improvement in mobility was observed, and $76 \%$ of the patients were found with intact repairs ${ }^{33}$. Another study of repair and augmentation of massive injuries found $85 \%$ of repairs to be intact, and another study concluded that surgeons who seek to maximize structural wound healing in massive ruptures susceptible to primary repair can consider augmentation with this allograft ${ }^{19,34}$.

\section{Superior capsular reconstruction}

The shoulder capsule is a static stabilizer of the glenohumeral articulation, and its function is not well known. Biomechanical studies suggest that defects at this level increase glenohumeral translation in all directions, mainly upward at $5^{\circ}$ and $30^{\circ}$ of abduction. Its reconstruction restitutes translation to physiological conditions ${ }^{4}$.

Superior capsular reconstruction is indicated for irreparable massive tears of the rotator cuff, which are defined as the inability to move the tendon to its insertion place 4 . In young, active patients with irreparable injuries, where a reverse shoulder arthroplasty is evidently not recommended, superior capsule reconstruction is a valid alternative ${ }^{20}$. The best results have been obtained in patients who present an intact subscapular muscle. Patients with Grade 3 or 4 of fatty infiltration may be considered as candidates as long as they have a conserved active external rotation, as well as preservation of subacromial space ${ }^{35}$. This procedure allows the restoration of the superior stability and muscular balance of the shoulder, improving articular function ${ }^{20}$.

The fact that autologous grafts $>35 \mathrm{~mm}$ in the anteroposterior direction may become stuck in the cannulas must be taken into consideration. Moreover, the force produced in an attempt to pass it through the cannula can 
unhinge the anchors positioned in the glenoid. This situation can be avoided using the zip-line technique, where the graft is pulled through an accessory portal while pushing the graft from each side using grasping forceps through the anchors of the glenoid. A modification is added to this procedure when dividing the cannula, to do so radially, which keeps the graft form getting stuck ${ }^{21}$.

Among its advantages are a low infection rate, restoration of a stable fulcrum of the shoulder, not hampering future use of a reverse shoulder arthroplasty, a lower cost compared to a reverse shoulder arthroplasty, and a chance to revert pseudoparalysis. Some of its disadvantages include the fact that it requires a longer rehabilitation time of compared to a reverse shoulder arthroplasty and a possibility of requiring a reverse arthroplasty as the next step in case of failure. Moreover, it is technically more demanding than a reverse arthroplasty and does not generally restore normal strength of the shoulder. Pseudoparalysis is not compensated so frequently with this method compared to a full repair of the rotator cuff $^{21}$.

Reported complications include infection, bleeding, wound dehiscence, pain, articular stiffness, deep venous thrombosis, pulmonary embolism, and death ${ }^{20}$.

Patients who undergo a superior capsule reconstruction clinically and statistically present an improvement in arc of motion, with a low risk of failure, complications or surgical reintervention, a decrease in pain registered on the visual analog scale from 3.5 to 1.4 , and an ASES score increase from 32.5 to 86.3. Arcs of motion showed an average arc of $153.3^{\circ}$ and an external rotation of $58.9^{\circ}$. Namdari registered that in healthy individuals, an anterior flexion of $121^{\circ}$ and an external rotation of $41.8^{\circ}$ are enough to carry out daily activities without difficulty 20,36 .

Mihata et al. studied clinical results in 24 shoulders with irreparable ruptures, where a fascia lata graft was utilized. Two years after the procedure, there was an improvement in rotator cuff function, as well as in muscle strength of the shoulder and an increase in the acromiohumeral distance of $4.1 \pm 1.7 \mathrm{~mm}$. There were no new ruptures or progression of muscle atrophy $y^{4,37}$.

\section{Subacromial spacer}

This is an innovative technique where a subacromial biodegradable spacer is used. It is placed between the acromion and the humeral head, allowing a soft glide without friction, thus restoring shoulder biomechanics ${ }^{22}$.

The system consists of placing a cannula and a spacer made of biodegradable materials in a period of
12 months, which begins to deflate after the $3^{\text {rd }}$ month $^{22,23}$. It is contraindicated in patients with allergies to the L-lactic-co- $\varepsilon$-caprolactone component, with latent infections or data of subacromial tissue necrosis ${ }^{22}$.

Its effects of symptomatic improvement last longer than the natural period of degradation of the implant. It is not understood why both pain and functionality continue to improve after the period of disintegration ${ }^{22,23}$.

Its use is recommended in patients with irreparable tears of the rotator cuff without glenohumeral arthrosis after 6 months of conservative management without response. It can be used to protect partial repairs ${ }^{22}$. Its risks include response to a foreign body, local irritation at the location of the wound, local infection and tissue necrosis, and an increase in pain and component displacement ${ }^{22}$.

In a systemic review conducted in 2018, exclusively including massive injuries considered irreparable for management with this system, confirmed during arthroscopy, with a symptomatology consisting of persistent pain and loss of function of the shoulder. The majority of authors agreed to have glenohumeral arthrosis as exclusion criteria, specifically a Hamada Grade $>3$. Pseudoparalysis and subscapular injuries were also considered as contraindications, the latter because of the possible anterior migration of the spacer. Mobility arcs improved considerably with an anterior elevation of $124^{\circ} \pm 48^{\circ}-147^{\circ} \pm 35^{\circ}$, abduction of $113 \pm 53^{\circ}-136^{\circ}$ $\pm 43^{\circ}$, and an external rotation of $32^{\circ} \pm 19^{\circ}-52^{\circ} \pm 25^{\circ}$. There was also an improvement in the visual analog scale from 7 to a 2.2 after surgery ${ }^{23}$.

Between 2014 and 2016, a total of 15 cases were studied, 3 of which had previous failed repairs. After applying the Constant score, no significant differences were found at 12 months nor in the QuickDASH score ${ }^{38}$. The QuickDASH is an abbreviated version of the DASH outcome measure with only 11 items instead of original 30 , to measure thoracic functions and symptoms of patients with disorders at this level ${ }^{39}$. These items are assessed, converting the results into a scale from 0 to $100^{40}$. The results showed a low success rate of $53.3 \%$. It was not possible to determine whether or not there is a specific group of patients that benefitted from this procedure in stiffness, pain as a predominant symptom or mild arthrosis in radiographic studies ${ }^{38}$.

\section{Conclusion}

The integration of procedures such as superior capsular reconstruction or subacromial balloons allows 
us to expand therapeutic options without causing additional injuries in soft tissue and preserving articulation. There are additional non-arthroscopic methods that can be considered in case of unsatisfactory results. Their implementation is not hampered by the application of the arthroscopic techniques mentioned above.

\section{Conflicts of interest}

The authors declare not to have any conflicts of interest; furthermore, they did not receive any sort of compensation for this.

\section{Ethical responsibilities}

People and animal protection. The authors declare that for this research, there were no experiments performed in humans or animals.

Data confidentiality. The authors declare to have followed all protocols of their work center about the publication of the data of the patients.

Privacy rights and informed consent. The authors declare that this article does not include data of any of the patients.

\section{References}

1. Dines DM, Moynihan DP, Dines JS, McCann P. Irreparable rotator cuff tears: what to do and when to do it; the surgeon's dilemma. J Bone Joint Surg Am. 2006;88:2294-302.

2. Herrmann SJ, Izadpanah K, Südkamp NP, Strohm PC. Tears of the rotator cuff. Causes-diagnosis-treatment. Acta Chir Orthop Traumato Cech. 2014:81:256-66.

3. Ogata S, Uhthoff HG. Acromial enthesopathy and rotator cuff tear. Clin Orthop Relat Res. 1990;254:39-48.

4. Greenspoon JA, Petri M, Warth, RJ, Millett PJ. Massive rotator cuff tears: pathomechanics, current treatment options, and clinical outcomes. J Shoulder Elbow Surg. 2015;24:1493-505.

5. Loew M, Habermeyer P, Wiedemann E, Rickert M, Gohlke F. Empfehlungen zu diagnostik und begutachtung der traumatischen rotatorenmanschettenläsion. Der Unfallchirurg. 2000;103:417-26.

6. Miller RH $3^{\text {rd }}$, Azar FM, Throckmorton TW. Shoulder and elbow injuries. In: Azar FM, Canale ST, Beaty JH, editors. Campbell's Operative Orthopaedics. Philadelphia, PA: Elsevier/Mosby; 2016. p. 2298-345.

7. Lädermann A, Burkhart SS, Hoffmeyer P, Neyton L, Collin P, Yates E, et al. Classification of full-thickness rotator cuff lesions: a review. EFORT Open Rev. 2016;1:420-30.

8. Patte D. Classification of rotator cuff lesions. Clin Orthop Relat Res. 1990;254:81-6.

9. Bateman JE. The diagnosis and treatment of ruptures of the rotator cuff. Surg Clin North Am. 1963;43:1523-30.

10. Ellman H, Kay SP, Wirth M. Arthroscopic treatment of full-thickness rotator cuff tears: 2-to 7-year follow-up study. Arthroscopy. 1993:9:195-200.

11. DeOrio JK, Cofield RH. Results of a second attempt at surgical repair of a failed initial rotator-cuff repair. J Bone Joint Surg Am. 1984;66:563-7.

12. Gerber C, Fuchs B, Hodler J. The results of repair of massive tears of the rotator cuff. J Bone Joint Surg Am. 2000;82:505-15.

13. Manaster BJ, May DA, Disler DG. Shoulder 2: soft tissues. In: Manaster BJ, May DA, Disler DG, editors. Musculoskeletal Imaging: the Requisites. USA, Philadelphia, PA: Elsevier/Mosby; 2013. p. 73-94.

14. Blankenbaker DG, Davis KW. Musculotendinous pathology. In: Blankenbaker DG, Davis KW, editors. Diagnostic Imaging: musculoskeletal Trauma. Philadelphia, PA: Elsevier; 2016. p. 92-157.
15. Goutallier D, Postel JM, Bernageau J, Lavau L, Voisin MC. Fatty muscle degeneration in cuff ruptures. Clin Orthop Relat Res. 1994;304:78-83.

16. Lippe J, Spang JT, Leger RR, Arciero RA, Mazzocca AD, Shea KP. Inter-rater agreement of the goutallier, patte, and warner classification scores using preoperative magnetic resonance imaging in patients with rotator cuff tears. Arthroscopy. 2012;28:154-9.

17. Hawi N, Schmiddem U, Omar M, Stuebig T, Krettek C, Petri M, et al. Arthroscopic debridement for irreparable rotator cuff tears. Open Orthop J. 2016;10:324-9.

18. Neer CS $2^{\text {nd }}$, Craig EV, Fukuda $H$. Cuff-tear arthropathy. J Bone Joint Surg Am. 1983:65:1232-44.

19. Richter DL, Brockmeier SF. Arthroscopic patch augmentation for rotator cuff repair. Tech Should Elb Surg. 2016;17:144-8.

20. Sochacki KR, McCulloch PC, Lintner DM, Harris JD. Superior capsular reconstruction for massive rotator cuff tear leads to significant improvement in range of motion and clinical outcomes: a systematic review. Arthroscopy. 2019;35:1269-77.

21. Burkhart SS, Denard PJ, Adams CR, Brady PC, Hartzler RU. Arthroscopic superior capsular reconstruction for massive irreparable rotator cuff repair. Arthrosc Tech. 2016;5:e1407-18.

22. Savarese E, Romeo R. New solution for massive, irreparable rotator cuff tears: the subacromial biodegradable spacer. Arthrosc Tech. 2012;1: e69-74.

23. Moon AS, Patel HA, Ithurburn MP, Brabston EW, Ponce BA, Momaya AM. Subacromial spacer implantation for the treatment of massive irreparable rotator cuff tears: a systematic review. Arthroscopy. 2019;35:607-14.

24. Boileau $P$, Baqué F, Valerio L, Ahrens $P$, Chuinard C, Trojani C. Isolated arthroscopic biceps tenotomy or tenodesis improves symptoms in patients with massive irreparable rotator cuff tears. J Bone Joint Surg Am. 2007;89:747-57.

25. Gartsman GM. Massive, irreparable tears of the rotator cuff. Results of operative debridement and subacromial decompression. J Bone Joint Surg Am. 1997;79:715-21.

26. Burrus $C$, Deriaz O, Luthi F, Konzelmann M. Role of pain in measuring shoulder strength abduction and flexion with the constant-murley score. Ann Phys Rehabil Med. 2017;60:258-62.

27. Vrotsou K, Ávila M, Machón M, Mateo-Abad M, Pardo Y, Garin O, et al. Constant-murley score: systematic review and standardized evaluation in different shoulder pathologies. Qual Life Res. 2018;27:2217-26.

28. Burkhart SS. Arthroscopic débridement and decompression for selected rotator cuff tears. Clinical results, pathomechanics, and patient selection based on biomechanical parameters. Orthop Clin North Am. 1993;24:111-23.

29. Zvijac JE, Levy HJ, Lemak LJ. Arthroscopic subacromial decompression in the treatment of full thickness rotator cuff tears: a 3- to 6-year follow-up. Arthroscopy. 1994:10:518-23.

30. Kempf JF, Gleyze P, Bonnomet F, Walch G, Molé D, Frank A, et al. A multicenter study of 210 rotator cuff tears treated by arthroscopic acromioplasty. Arthroscopy. 1999;15:56-66.

31. Cuff DJ, Pupello DR, Santoni BG. Partial rotator cuff repair and biceps tenotomy for the treatment of patients with massive cuff tears and retained overhead elevation: midterm outcomes with a minimum 5 years of follow-up. J Shoulder Elbow Surg. 2016;25:1803-9.

32. Oh JH, McGarry MH, Jun BJ, Gupta A, Chung KC, Hwang J, et al. Restoration of shoulder biomechanics according to degree of repair completion in a cadaveric model of massive rotator cuff tear: importance of margin convergence and posterior cuff fixation. Am J Sports Med. 2012;40:2448-53.

33. Gupta AK, Hug K, Berkoff DJ, Boggess BR, Gavigan M, Malley PC, et al. Dermal tissue allograft for the repair of massive irreparable rotator cuff tears. Am J Sports Med. 2012;40:141-7.

34. Ferguson DP, Lewington MR, Smith TD, Wong IH. Graft utilization in the augmentation of large-to-massive rotator cuff repairs: a systematic review. Am J Sports Med. 2016;44:2984-92.

35. Noyes MP, Denard PJ. Arthroscopic superior capsular reconstruction: indications and outcomes. Oper Tech Sports Med. 2018;26:29-34.

36. Namdari S, Yagnik G, Ebaugh DD, Nagda S, Ramsey ML, Williams GR Jr., et al. Defining functional shoulder range of motion for activities of daily living. J Shoulder Elbow Surg. 2012;21:1177-83.

37. Mihata T, Lee TQ, Watanabe C, Fukunishi K, Ohue M, Tsujimura T, et al. Clinical results of arthroscopic superior capsule reconstruction for irreparable rotator cuff tears. Arthroscopy. 2013;29:459-70.

38. Lorente A, Ruiz MA, Ruiz R, Vega, M, Álvarez R, Paniagua A, et al. Malos resultados a corto plazo del balón subacromial InSpace®. Resultados de 15 casos consecutivos con un año de seguimiento. Rev Esp Artrosc Cir Articul. 2017;24:197-203.

39. Southam M, Driessens S, Burton C, Pope R, Thurnwald P. A retrospective cohort study of QuickDASH scores for common acute trauma conditions presenting for hand therapy. J Hand Ther. 2017;30:41-8.

40. Franchignoni F, Vercelli S, Giordano A, Sartorio F, Bravini E, Ferriero G Minimal clinically important difference of the disabilities of the arm, shoulder and hand outcome measure (DASH) and its shortened version (QuickDASH). J Orthop Sports Phys Ther. 2014;44:30-9. 